\title{
THE MINIMAL NUMBERS OF BINARY FORMS ${ }^{1}$
}

\section{RUFUS OLDENBURGER AND ARTHUR PORGES}

1. Introduction. One of us proved that for certain fields $K$ a form $F$ of degree $m$ can be written as a linear combination of $m$ th powers of linear forms. Such a combination is termed a representation of $F$ and the least possible number of terms in any such representation is called the minimal number of $F$ with respect to $K$. The minimal number depends on both $F$ and $K$. For fields $K$ with characteristic greater than $n$, and binary forms $F$ of degree $n$, it has been proved ${ }^{2}$ that the minimal number ranges over at least $1,2, \cdots, n$, and at most $1,2, \cdots, n+1$, but the exact range was not determined. In the present paper the authors prove that the range is precisely $1,2, \cdots, n$.

2. Preliminary lemmas. In what follows we use identity of polynomials in the usual sense, namely polynomials $P$ and $Q$ are identical if the coefficients of $P$ equal the corresponding coefficients of $Q$.

Since the order of a field $K$ is greater than $m$ if the characteristic of $K$ is greater than $m$, we have the following lemma.

Lemma 1. For a field $K$ with characteristic greater than $m$ a polynomial $P$ of degree $m$ is equal to a polynomial $Q$ for all values of the variables if and only if $P$ and $Q$ are identical.

An immediate consequence of Lemma 1 is the following lemma.

Lemma 2. For a field $K$ with characteristic greater than $m$, a polynomial $P$ of degree $m$ not identically zero is different from zero for at least one set of values of the variables.

Lemma 3. Let $K$ be a field with characteristic greater than $m$. Let $\Delta$ be the determinant

$$
\Delta=\left|\begin{array}{cccc}
1 & \cdots & 1 & b_{1} \\
a_{1} & \cdots & a_{m} & b_{2} \\
\cdot & \cdots & c^{m} & \cdot \\
a_{1}^{m} & \cdots & a_{m}^{m} & b_{m+1}
\end{array}\right|
$$

of order $m+1, m \geqq 1$, with elements in $K$, and suppose that the $b$ 's are not all zero. The determinant $\Delta$ is not identically zero in the a's.

\footnotetext{
1 Presented to the Society, April 13, 1940.

2 R. Oldenburger, Polynomials in several variables, Annals of Mathematics, (2), vol. 41 (1940), no. 3, pp. 694-710.
} 
Lemma 3 is valid when $m=1$. For $m \geqq 2$ we have the following sequence of equalities:

$$
\begin{aligned}
& \Delta=\left|\begin{array}{ccccc}
1 & 0 & \cdots & 0 & b_{1} \\
a_{1} & \left(a_{2}-a_{1}\right) & \cdots & \left(a_{m}-a_{1}\right) & b_{2} \\
a_{1}^{2} & \left(a_{2}^{2}-a_{1}^{2}\right) & \cdots & \left(a_{m}^{2}-a_{1}^{2}\right) & b_{3} \\
\cdot & \cdot & \cdots & \cdot & \cdot \\
a_{1}^{m} & \left(a_{2}^{m}-a_{1}^{m}\right) & \cdots & \left(a_{m}^{m}-a_{1}^{m}\right) & b_{m+1}
\end{array}\right| \\
& =\left(a_{2}-a_{1}\right) \cdots\left(a_{m}-a_{1}\right)\left|\begin{array}{ccccc}
1 & 0 & \cdots & 0 & b_{1} \\
0 & 1 & \cdots & \left(b_{2}-b_{1} a_{1}\right) \\
0 & a_{2} & \cdots & a_{m} & \left(b_{3}-b_{2} a_{1}\right) \\
\cdot & \cdot & \cdots & \cdot \\
0 & a_{2}^{m-1} & \cdots & a_{m}^{m-1} & \left(b_{m+1}-b_{m} a_{1}\right)
\end{array}\right| .
\end{aligned}
$$

Thus

$$
\Delta=\left(a_{2}-a_{1}\right) \cdots\left(a_{m}-a_{1}\right)\left(M-a_{1} N\right),
$$

where

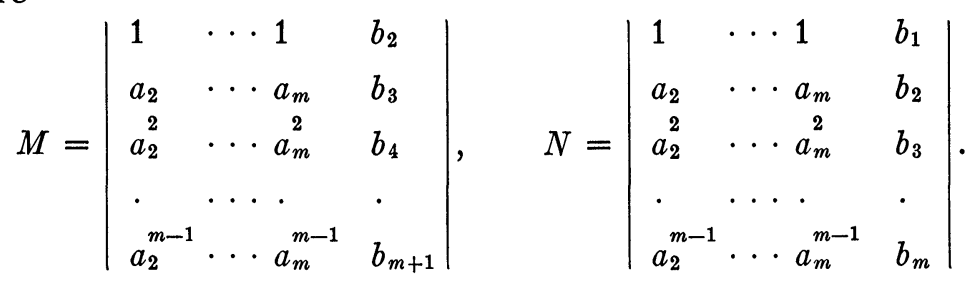

The lemma now follows by induction.

It is to be remarked that if the characteristic of a field $K$ is greater than $m$, each binary form of degree $m$ with coefficients in $K$ can be written as

$$
b_{1} x^{m}+m b_{2} x^{m-1} y+\cdots+b_{m+1} y^{m},
$$

where the coefficient of $b_{i} x^{m-i+1} y^{i-1}$ is the binomial coefficient of $x^{m-i+1} y^{i-1}$ in the expansion of $(x+y)^{m}$.

3. The range of the minimal number. We proceed with the following theorem in which $I(m)$ denotes the integer

$$
\frac{1}{2} m[m(m-1)+2] \text {. }
$$

Theorem 1. For a field $K$ with characteristic greater than $m$, and order at least $I(m)$, the minimal number of a binary form $F$ does not exceed $m$. 
We write $F$ as in (4). We exclude the trivial case $F \equiv 0$. If $b_{1} \neq 0$ while $b_{i}=0$ for $i>1$, the form $F$ is simply $b_{1} x^{m}$, whence the minimal number of $F$ is 1 ; similarly, if $b_{m+1} \neq 0$ while $b_{i}=0$ for $i<m+1$. If $m \geqq 2$ while $b_{1}$ and $b_{m+1}$ are not zero, and if further $b_{i}=0$ for $i=2,3, \cdots, m$, the form $F$ is nonsingular in the sense of Oldenburger, ${ }^{3}$ and has minimal number 2 .

If $F$ is of the first degree, the minimal number of $F$ is clearly 1 , while if $F$ is quadratic, the minimal number is identical ${ }^{4}$ with the rank of $F$ and does not exceed 2 .

It remains to consider forms $F$ of degree at least 3 for which $b_{2}, \cdots, b_{m}$ are not all zero. It is no restriction to assume that $b_{m+1} \neq 0$. For if $b_{m+1}=0$, the form $F$ can be transformed nonsingularly into a form with this property, as is clear from the following argument. We write $F=F(x, y)$. Since $F \not \equiv 0$, there exist values $a$ and $b$ of $x$ and $y$ respectively such that $F(a, b) \neq 0$. We make the transformation

$$
x=x^{\prime}+a y^{\prime}, \quad y=x^{\prime}+b y^{\prime},
$$

on $F$ to obtain a form $F^{\prime}\left(x^{\prime}, y^{\prime}\right)$. Evidently, $F^{\prime}(0,1)=F(a, b)$.

We consider the following equality:

$$
b_{1} x^{m}+m b_{2} x^{m-1} y+\cdots+b_{m+1} y^{m}=\sum_{i=1}^{m} \lambda_{i}\left(x+a_{i} y\right)^{m} .
$$

The equality (5) is identically satisfied if the $\lambda$ 's and $a$ 's are chosen so that the following system of linear equations is valid:

$$
\begin{gathered}
\lambda_{1}+\lambda_{2}+\cdots+\lambda_{m}=b_{1}, \\
a_{1} \lambda_{1}+a_{2} \lambda_{2}+\cdots+a_{m} \lambda_{m}=b_{2}, \\
a_{1}^{2} \lambda_{1}+a_{2}^{2} \lambda_{2}+\cdots+a_{m}^{2} \lambda_{m}=b_{3}, \\
\cdot \cdot \cdot \cdot \cdot \cdot \cdot \cdot \cdot \cdot \cdot \cdot \cdot \cdot, \\
{ }_{a_{1}^{m} \lambda_{1}}+a_{2}^{m} \lambda_{2}+\cdots+a_{m}^{m} \lambda_{m}=b_{m+1} .
\end{gathered}
$$

We shall prove that the $a$ 's can be chosen so that the rank of the matrix of coefficients of the $\lambda$ 's in (6) is $m$, while the rank of the augmented matrix is also $m$. The determinant of the augmented matrix of (6) is $\Delta$. We write $\Delta$ as in (2).

In the expansion of $M$, the terms of lowest degree $\alpha$ in the $a$ 's are those which have $b_{m+1}$ as coefficient. The degree $\alpha$ is explicitly

${ }^{3} \mathrm{R}$. Oldenburger, Rational equivalence of a form to a sum of pth powers, Transactions of this Society, vol. 44 (1938), pp. 219-249.

${ }^{4}$ M. Bôcher, Introduction to Higher Algebra, p. 135. 
$\frac{1}{2}(m-1)(m-2)$. The terms of lowest degree, in the expansion of the polynomial $a_{i} N$ for any $i$, are at least of degree $\alpha+1$. Thus the terms of degree $\alpha$ in the polynomial $M-a_{i} N[i$ in the range $2,3, \cdots, m]$ arise from $M$ only, whence $M-a_{i} N$ is not identically zero in the $a$ 's. Thus the polynomial

$$
\left(M-a_{2} N\right) \cdots\left(M-a_{m} N\right) N
$$

of degree at most $I(m)-1$ is not identically zero in the $a$ 's. Evidently, $N \neq 0$ implies that $a_{2}, \cdots, a_{m}$ are distinct. Choose a set of values of $a_{2}, \cdots, a_{m}$ such that the polynomial (7) is different from zero. For this choice of the $a$ 's the polynomial $M-a_{1} N$ is linear in $a_{1}$, and $M-a_{1} N=0$ has a solution for $a_{1}$, distinct from $a_{2}, \cdots, a_{m}$. Thus there exist mutually distinct values of $a_{1}, a_{2}, \cdots, a_{m}$, such that $\Delta=0$. Since the $a$ 's are mutually distinct, the matrix of coefficients of the $\lambda$ 's in (6) has rank $m$. It follows that the augmented matrix has the same rank. It is well known that a system of linear equations has a solution if and only if the matrix of coefficients and the augmented matrix have the same rank. ${ }^{5}$ Theorem 1 is now proved.

The following theorem was proved by Oldenburger. ${ }^{6}$

THEOREM 2. For a field with characteristic greater than $m$, the minimal number of $x^{m-1} y$ is $m$.

If the sum $\lambda_{i} L_{i}^{m}(i=1,2, \cdots, m)$ is chosen to be a minimal representation of $x^{m-1} y$, the sum

$$
\lambda_{1} L_{1}^{m}+\cdots+\lambda_{\rho} L_{\rho}^{m}, \quad \rho \leqq m,
$$

is minimal. Thus for each $\rho$ in the range $1,2, \cdots, m$ there is a binary form of degree $m$ with minimal number $\rho$. Applying Theorem 1 we have arrived at the following result.

Theorem 3. Let a field $K$ be given as in Theorem 1. For the field $K$ the range of the minimal numbers of binary forms of degree $m$ is $1,2, \cdots, m$.

Armour Institute of Technology

${ }^{5}$ M. Bôcher, Introduction to Higher Algebra, p. 46.

${ }^{6}$ See first reference to Oldenburger above. 